Review began 02/13/2022 Review ended 02/20/2022 Published 02/24/2022

๑) Copyright 2022

Al Ghadeer et al. This is an open access article distributed under the terms of the Creative Commons Attribution License CC BY 4.0., which permits unrestricted use, distribution, and reproduction in any medium, provided the original author and source are credited.

\section{Vitamin D Serum Levels in Type 2 Diabetic Patients: A Cross-Sectional Study}

Hussain A. Al Ghadeer ${ }^{1}$, Mohammed S. AlRamadan ${ }^{2}$, Mohammed M. Al Amer ${ }^{3}$, Meshal J. Alshawaf ${ }^{2}$, Fatimah J. Alali ${ }^{4}$, Aisha A. Bubshait ${ }^{2}$, Maryam A. Alramadhan ${ }^{5}$, Zainab Almurayhil ${ }^{6}$, Nasser S. Aldandan 2 , Mohammed A. AlKhamis ${ }^{2}$, Habeeb A. AlHaddad ${ }^{2}$, Abdulatif AlOmair ${ }^{7}$

1. Paediatrics, Maternity and Children Hospital, AlAhsa, SAU 2. Internal Medicine, King Faisal University, AlAhsa, SAU 3. Internal Medicine, College of Medicine, King Faisal University, AlAhsa, SAU 4. Medicine, king Faisal University, AlAhsa, SAU 5. College of Medicine, King Faisal University, AlAhsa, SAU 6. Medicine, King Faisal University, AlAhsa, SAU 7. Internal Medicine, King Faisal University, AlHofuf, SAU

Corresponding author: Hussain A. Al Ghadeer, hu.alghadeer@gmail.com

\section{Abstract \\ Background and objective}

Type 2 diabetes mellitus (T2DM) is a chronic metabolic disorder characterized by hyperglycemia. It is linked with an increase in morbidity (e.g., blindness, kidney failure, stroke, cardiovascular diseases, limb amputations), premature mortality, high healthcare costs, and is quickly becoming a global epidemic disorder. Several studies have shown that vitamin D supplements reduce insulin resistance in T2DM and improve insulin secretion and sensitivity. In this study, we aimed to determine the prevalence of vitamin D deficiency in T2DM patients in Saudi Arabia.

\section{Methods}

This was a retrospective cross-sectional study conducted at the King Faisal University Health Centre in Saudi Arabia. The study used patient data during the period from October 2014 to January 2021. After obtaining approval from the King Faisal University Polyclinic Administration, we collected patient data from the King Faisal University Health Centre. The Ethics and Research Committee at the College of Medicine of King Faisal University granted ethical approval with the approval number (2020-11-82). The relevant patient data were collected, including age, gender, nationality, and blood test findings (vitamin D and HbA1c levels).

\section{Results}

A total of 191 T2DM patients participated in this study. The mean age of the patients was $56.1 \pm 11.4$ years (range: $21-85$ years); 107 (56\%) patients were females, and 137 (71.7\%) were Saudis. There were 134 (70.2\%) patients with vitamin D deficiency, 53 (27.7\%) with vitamin D insufficiency, and only four (2.1\%) with normal vitamin D levels.

\section{Conclusion}

Based on our findings, the prevalence of vitamin D deficiency among T2DM patients is highly associated with poor diabetic control.

Categories: Internal Medicine

Keywords: 25 (oh) vitamin d, cross sectional studies, interna medicine, saudi arabia. ， diabetes type ii

\section{Introduction}

Type 2 diabetes mellitus (T2DM) is a chronic metabolic disorder characterized by hyperglycemia [1]. It is associated with a significant increase in morbidity (e.g., blindness, kidney failure, stroke, cardiovascular diseases, limb amputations), premature mortality, high healthcare costs, and is rapidly becoming an epidemic disorder of global proportions [2]. The World Health Organization (WHO) predicts that the number of diabetic patients worldwide will exceed 370 million by 2030 [3]. Locally, Saudi Arabia ranks first among Middle Eastern countries in terms of estimated diabetes mellitus cases [4]. Furthermore, various epidemiological studies show that the prevalence of diabetes mellitus is increasing every year, paralleling the increase in life expectancy, as the elderly population is at a higher risk of developing T2DM [5]. Also, multiple studies show that more than $90 \%$ of T2DM cases are strongly associated with poor lifestyle, obesity $\left(\right.$ BMI $\left.>30 \mathrm{~kg} / \mathrm{m}^{2}\right)$, and reduced physical activity $[4,5]$.

Vitamin D is an essential nutrient for humans, which can be obtained both exogenously and endogenously. The primary source of vitamin D is endogenous synthesis by the skin with the help of ultraviolet light [5]. Vitamin D deficiency is indicated if the serum level of 25-hydroxyvitamin D (25(OH)D) falls below the level 
of $50 \mathrm{nmol} / \mathrm{L}$, while serum concentrations $<75 \mathrm{nmol} / \mathrm{L}$ are indicative of vitamin D insufficiency $[2,4]$. According to WHO, more than one billion people are vitamin D-deficient or insufficient worldwide [4,5]. Furthermore, multiple studies estimate that the global prevalence of vitamin D deficiency and insufficiency is between 30 and $87 \%$ [6]. Several factors, including genetics, lifestyle-related, environmental, and nutritional, all play a role in the development of vitamin D deficiency and insufficiency [1]. Additionally, an increase in BMI increases the risk of developing vitamin D insufficiency and deficiency because vitamin D is deposited in adipose tissue and becomes biologically inactive [2]. Furthermore, due to the increased need for vitamin D in certain patient groups, these patient groups, such as infants, lactating women, and adolescents, are at a higher risk of developing vitamin D deficiency and insufficiency [2].

According to several studies, vitamin D plays an important role in the prevention of cardiovascular diseases and cancers, inhibition of parathyroid hormone secretion and adaptive immunity, and the promotion of innate immunity $[2,7]$. Furthermore, vitamin D is primarily involved in glycemic control and the prevention of diabetic complications [1]. Several observational and cross-sectional studies [2,6] have suggested a link between vitamin D deficiency and the development of metabolic syndrome or T2DM and cardiovascular diseases. Vitamin D promotes insulin action by regulating insulin receptor gene expression, which increases insulin sensitivity [2]. Accordingly, vitamin D deficiency is likely to cause insulin resistance, which is the main reason for the development of T2DM $[1,8]$. Furthermore, insulin secretion is calcium-dependent, and a lack of vitamin D impairs glucose-mediated insulin release [2]. Several studies have shown that vitamin D supplements reduce insulin resistance in T2DM and improve insulin secretion and sensitivity [3,4].

The currently available data in the literature is insufficient to support the link between T2DM and vitamin D deficiency, as large trials are required for the same. In light of this, the goal of this study was to determine the prevalence of vitamin D deficiency in type 2 diabetic patients in Saudi Arabia.

\section{Materials And Methods}

\section{Study design}

This was a retrospective cross-sectional study conducted at the King Faisal University Health Centre in Saudi Arabia. The study collected patient data from October 2014 to January 2021.

\section{Procedure}

Following approval from the King Faisal University Polyclinic Administration, we collected patient data from the King Faisal University Health Centre. The Ethics and Research Committee, College of Medicine, King Faisal University granted the ethical approval (approval number: 2020-11-82). The relevant patient data were gathered, including age, gender, nationality, and blood test results (vitamin D and HbA1c levels).

\section{Inclusion and exclusion criteria}

The inclusion criteria were as follows: patients aged more than 18 years old, diagnosed with T2DM >3 months ago, and followed up regarding DM on a regular basis at the King Faisal University Health Centre.

The exclusion criteria were as follows: patients aged $<18$ years, those who were recently diagnosed with T2DM, patients diagnosed with DM type I, patients presenting with no comorbid diseases related to abnormal lipids profile, e.g., endocrine, renal, or hepatic disorders.

\section{Research population}

A total of 191 T2DM patients who were one year or older were included in our study.

\section{Materials}

The cut-off values for the HbA1c level were from 4 to $5.9 \%$. The cut-off levels of vitamin D level were divided into the following ranges - normal: $50 \%-70 \mathrm{ng} / \mathrm{ml}$, insufficient: 30-49 ng/ml, and deficient: <30 ng/ml.

\section{Data analysis}

Two-tailed tests were used for all statistical analyses. A p-value of less than 0.05 was considered statistically significant. All variables, including patients' demographic data, HbA1c, and vitamin D level, were subjected to descriptive analysis based on the frequency and percentage distribution. The lipid profiles were shown

as means with standard deviations. The Pearson $\mathrm{X}^{2}$ test and exact probability test for small frequency distributions were used to test the relationship between vitamin D levels and type 2 diabetic patients' demographic data and HbA1c levels. Next, correlation analysis was used to determine the relationship between vitamin D levels and the patients' lipid profiles and HbA1c levels, using SPSS Statistics version 22 (IBM, Armonk, NY).

\section{Results}




\section{Cureus}

A total of $191 \mathrm{~T} 2 \mathrm{DM}$ were included in the study. The mean age of the patients was $56.1 \pm 11.4$ years (range: 21-85 years). The cohort comprised 107 (56\%) females and 137 (71.7\%) Saudis (Table 1 ).

\begin{tabular}{|c|c|c|}
\hline Variables & $\mathbf{N}$ & $\%$ \\
\hline \multicolumn{3}{|c|}{ Age group, years } \\
\hline$<40$ & 17 & $8.9 \%$ \\
\hline $40-59$ & 96 & $50.3 \%$ \\
\hline 60+ & 78 & $40.8 \%$ \\
\hline \multicolumn{3}{|l|}{ Gender } \\
\hline Male & 84 & $44.0 \%$ \\
\hline Female & 107 & $56.0 \%$ \\
\hline \multicolumn{3}{|l|}{ Nationality } \\
\hline Saudi & 137 & $71.7 \%$ \\
\hline Non-Saudi & 54 & $28.3 \%$ \\
\hline
\end{tabular}

TABLE 1: Demographic data of the patients

A total of 134 (70.2\%) patients were vitamin D-deficient, 53 (27.7\%) had insufficient vitamin D levels, and only four (2.1\%) had normal vitamin D levels (Figure 1).

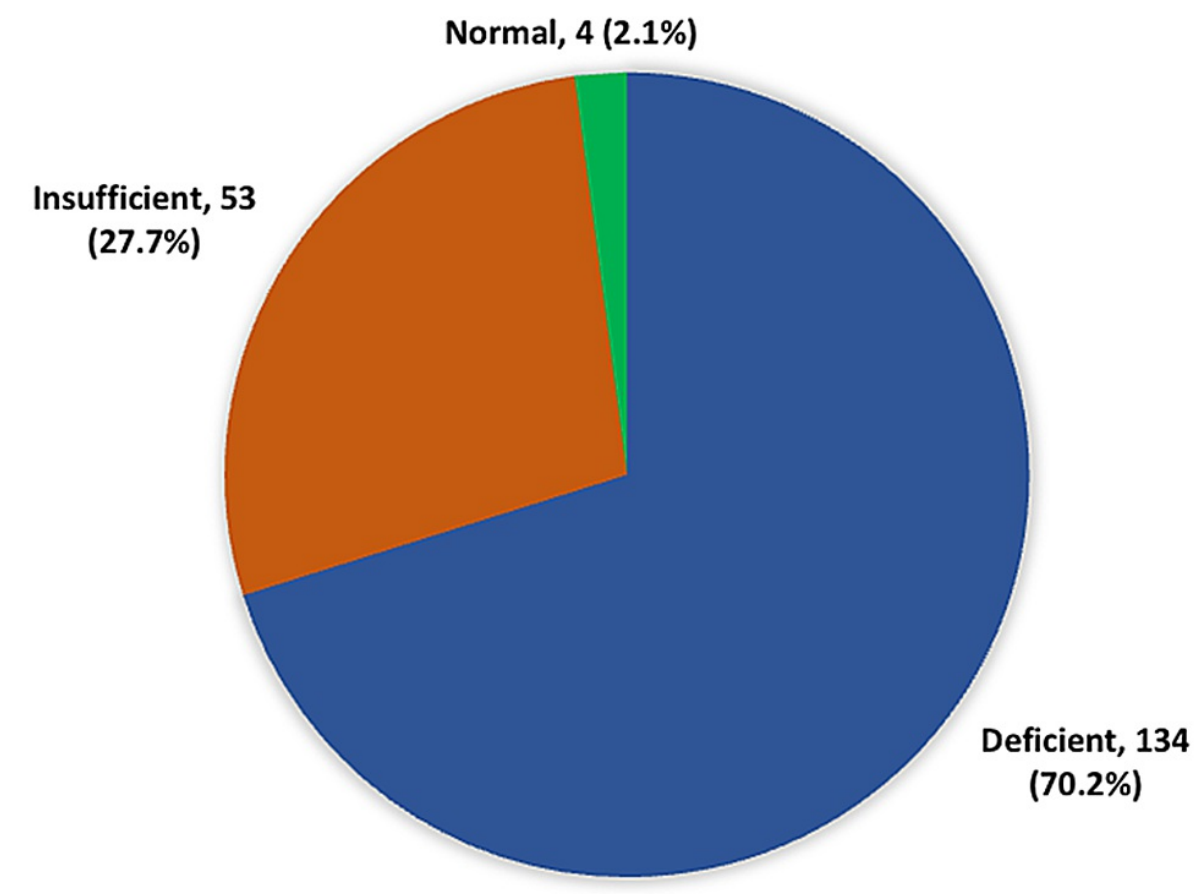

FIGURE 1: Vitamin D deficiency among type 2 diabetic patients

Of note, 178 (93.2\%) presented with HbA1c levels >5.9\%, and 13 (6.8\%) presented with HbA1c levels $<5.9 \%$ (Figure 3). 


\section{Cureus}

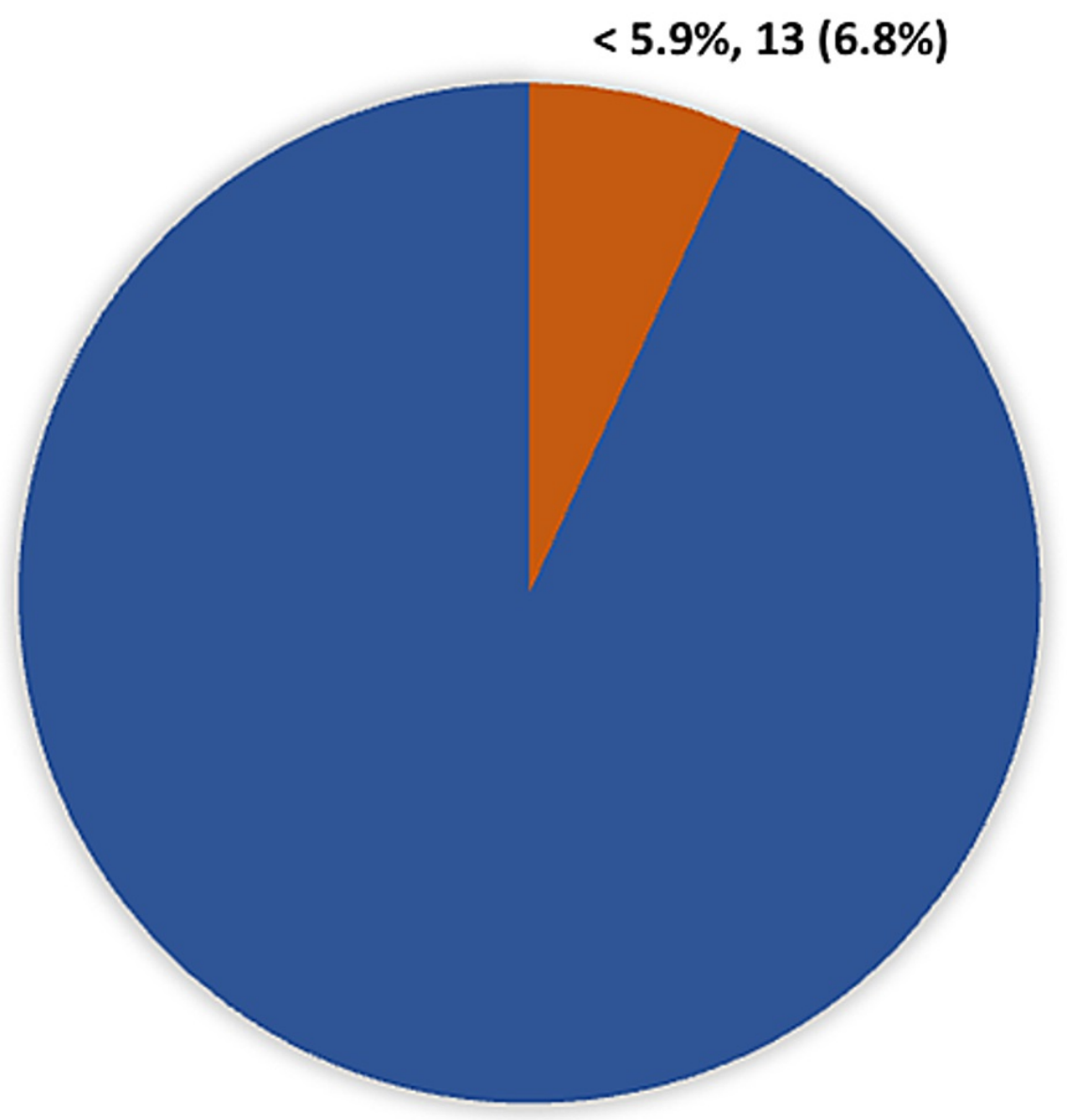

$>5.9 \%, 178(93.2 \%)$

FIGURE 2: HbA1c levels among type 2 diabetic patients

Vitamin D deficiency was detected among $76.5 \%$ of patients aged less than 40 years, compared to $60.3 \%$ of those aged 60 years or more; however, this difference had no statistical significance $(\mathrm{p}=0.162)$. Also, $71.4 \%$ of male patients had presented with vitamin D deficiency versus $69.2 \%$ of females, again with no significant difference $(\mathrm{p}=0.894)$. On the other hand, $71.9 \%$ of diabetic patients with $\mathrm{HbA1c}$ above $5.9 \%$ had presented with vitamin D deficiency in comparison to $46.2 \%$ of those with HbA1c levels less than $5.9 \%$, and this difference was statistically significant $(\mathrm{p}=0.048)$ (Table 2). 


\section{Cureus}

\begin{tabular}{|c|c|c|c|c|c|c|c|}
\hline \multirow{3}{*}{ Factors } & \multicolumn{6}{|c|}{ Vitamin D level } & \multirow{3}{*}{ P-value* } \\
\hline & \multicolumn{2}{|c|}{ Deficient } & \multicolumn{2}{|c|}{ Insufficient } & \multicolumn{2}{|c|}{ Normal } & \\
\hline & $\mathrm{N}$ & $\%$ & $\mathrm{~N}$ & $\%$ & $\mathrm{~N}$ & $\%$ & \\
\hline \multicolumn{8}{|c|}{ Age group, years } \\
\hline$<40$ & 13 & $76.5 \%$ & 4 & $23.5 \%$ & 0 & $0.0 \%$ & \multirow{3}{*}{$0.162^{\$}$} \\
\hline 40-59 & 74 & $77.1 \%$ & 20 & $20.8 \%$ & 2 & $2.1 \%$ & \\
\hline $60+$ & 47 & $60.3 \%$ & 29 & $37.2 \%$ & 2 & $2.6 \%$ & \\
\hline \multicolumn{8}{|l|}{ Gender } \\
\hline Male & 60 & $71.4 \%$ & 22 & $26.2 \%$ & 2 & $2.4 \%$ & \multirow[t]{2}{*}{0.894} \\
\hline Female & 74 & $69.2 \%$ & 31 & $29.0 \%$ & 2 & $1.9 \%$ & \\
\hline \multicolumn{8}{|c|}{ HbA1c level } \\
\hline$<5.9 \%$ & 6 & $46.2 \%$ & 7 & $53.8 \%$ & 0 & $0.0 \%$ & \multirow[t]{2}{*}{$0.048^{* \star \$}$} \\
\hline$>5.9 \%$ & 128 & $71.9 \%$ & 46 & $25.8 \%$ & 4 & $2.2 \%$ & \\
\hline
\end{tabular}

TABLE 2: Distribution of vitamin D deficiency by patients' personal data and HbA1c levels

${ }^{*}$ Pearson $\mathrm{X}^{2}$ test. ${ }^{\$}$ Exact probability test. ${ }^{* *} \mathrm{P}<0.05$ (significant)

\section{Discussion}

Many diseases, including T2DM, are linked with vitamin D deficiency [9]. Whether this relationship is causal or confounding is still a matter of debate. The active metabolite $1 \alpha, 25$-dihydroxy vitamin $\mathrm{D} 3(1,25(\mathrm{OH}) 2 \mathrm{D} 3)$ affects pancreatic $\beta$ cells and insulin secretion, and besides other factors, it may influence insulin sensitivity [10]. The vitamin D receptor (VDR) is present in many organs, which explains the fact that vitamin D metabolites may show numerous extra-skeletal effects [11]. A link exists between vitamin D deficiency and insulin resistance due to inflammation, as vitamin D deficiency is accompanied by higher inflammatory markers [12]. Nevertheless, a Mendelian randomization study on vitamin D and C-reactive protein failed to indicate a causal relationship [13].

The current study sought to assess vitamin D deficiency among type 2 diabetic patients in King Faisal University Health Centre in Saudi Arabia. The results showed that about one-fifth of the patients presented with vitamin D insufficiency, and slightly less than three-quarters presented with vitamin D deficiency (70.2\%), meaning nearly all cases presented with either vitamin D deficiency or insufficiency. These findings are consistent with those of Alhumaidi et al., who found that $98.5 \%$ of type 2 diabetic patients presented with 25-OH vitamin D deficiency [3]. The mean serum 25-OH vitamin D levels in the diabetic group were $15.7+7.5 \mathrm{ng} / \mathrm{mL}$. Al-Zahrani et al. reported that $98.4 \%$ of type 2 diabetic patients presented with vitamin D deficiency [14]. Nearly $75 \%$ of female diabetic patients presented with vitamin D deficiency compared to less than half of male diabetic patients (46.9\%), but $50.8 \%$ and $25.6 \%$ presented with vitamin D insufficiency. Bajaj et al. found that vitamin D deficiency $(<20 \mathrm{ng} / \mathrm{ml})$ was detected in $59.5 \%$ of type 2 diabetic patients, and their mean vitamin D level was $19.046 \pm 6.614 \mathrm{ng} / \mathrm{ml} \mathrm{[15].}$

In addition, many other studies have found a link between vitamin D deficiency and T2DM. The MiniFinland Health Survey evaluated T2DM subjects and found a significant negative association between serum vitamin D levels and the risk of T2DM [16]. Vitamin D deficiency was found in $91.1 \%$ of diabetic subjects in India, according to Daga et al. Approximately $60 \%$ of diabetic cases presented with high vitamin D deficiency, compared to $8.3 \%$ of controls [17]. In the current study, patients with poor diabetic control presented with the highest level of vitamin D deficiency, with nearly three-quarters of those with HbA1c greater than $5.9 \%$ presenting with vitamin D deficiency compared to less than half of those with HbA1c less than $5.9 \%$. This can be explained by the reported link between vitamin D deficiency and insulin resistance among diabetic patients. Many studies have assessed the link between vitamin $\mathrm{D}$ and the physiological function of the pancreatic $\beta$ cell as $\beta$ cells express VDRs, and 1a-hydroxylase is assessed in pancreatic tissue, equivalent with the expression of insulin [18]. Insulin secretion depends on calcium level, and it has been noted that vitamin D deficiency prevents glucose-facilitated insulin secretion $[18,10]$. Supplementation with vitamin D enhances insulin secretion based on oral glucose levels, with an increase in serum calcium and a decrease in free fatty acids [17]. 


\section{Conclusions}

The current study found that nearly all cases of T2DM presented with either vitamin D deficiency or insufficiency, with nearly one out of three patients presenting with vitamin D deficiency. Furthermore, vitamin D deficiency is linked to poor diabetic control due to a reported association with increased insulin resistance or a defect in insulin release.

\section{Additional Information \\ Disclosures}

Human subjects: Consent was obtained or waived by all participants in this study. Ethics and Research Committee, College of Medicine, King Faisal University issued approval 2020-11-82. Animal subjects: All authors have confirmed that this study did not involve animal subjects or tissue. Conflicts of interest: In compliance with the ICMJE uniform disclosure form, all authors declare the following: Payment/services info: All authors have declared that no financial support was received from any organization for the submitted work. Financial relationships: All authors have declared that they have no financial relationships at present or within the previous three years with any organizations that might have an interest in the submitted work. Other relationships: All authors have declared that there are no other relationships or activities that could appear to have influenced the submitted work.

\section{References}

1. Sheth JJ, Shah A, Sheth FJ, et al.: Does vitamin D play a significant role in type 2 diabetes? . BMC Endocr Disord. 2015, 15:5. 10.1186/s12902-015-0003-8

2. Ozfirat Z, Chowdhury TA: Vitamin D deficiency and type 2 diabetes . Postgrad Med J. 2010, 86:18-25. 10.1136/pgmj.2009.078626

3. Alhumaidi M, Agha A, Dewish M: Vitamin D deficiency in patients with type-2 diabetes mellitus in southern region of Saudi Arabia. Maedica (Bucur). 2013, 8:231-6.

4. Lee JM, Smith JR, Philipp BL, Chen TC, Mathieu J, Holick MF: Vitamin D deficiency in a healthy group of mothers and newborn infants. Clin Pediatr (Phila). 2007, 46:42-4. 10.1177/0009922806289311

5. Holick MF, Chen TC: Vitamin D deficiency: a worldwide problem with health consequences . Am J Clin Nutr. 2008, 87:1080S-6S. 10.1093/ajcn/87.4.1080S

6. Mauss D, Jarczok MN, Hoffmann K, Thomas GN, Fischer JE: Association of vitamin D levels with type 2 diabetes in older working adults. Int J Med Sci. 2015, 12:362-8. 10.7150/ijms.10540

7. McGreevy C, Williams D: New insights about vitamin D and cardiovascular disease: a narrative review . Ann Intern Med. 2011, 155:820-6. 10.7326/0003-4819-155-12-201112200-00004

8. Talaei A, Mohamadi M, Adgi Z: The effect of vitamin D on insulin resistance in patients with type 2 diabetes . Diabetol Metab Syndr. 2013, 5:8. 10.1186/1758-5996-5-8

9. Berridge MJ: Vitamin D deficiency and diabetes. Biochem J. 2017, 474:1321-32. 10.1042/BCJ20170042

10. Mathieu C, Gysemans C, Giulietti A, Bouillon R: Vitamin D and diabetes. Diabetologia. 2005, 48:1247-57. 10.1007/s00125-005-1802-7

11. Bouillon R, Carmeliet G, Verlinden L, et al.: Vitamin D and human health: lessons from vitamin D receptor null mice. Endocr Rev. 2008, 29:726-76. 10.1210/er.2008-0004

12. Kolb H, Mandrup-Poulsen T: An immune origin of type 2 diabetes? . Diabetologia. 2005, 48:1038-50. 10.1007/s00125-005-1764-9

13. Liefaard MC, Ligthart S, Vitezova A, et al.: Vitamin D and C-reactive protein: a Mendelian randomization study. PLoS One. 2015, 10:e0131740. 10.1371/journal.pone.0131740

14. Al-Zahrani MK: The prevalence of vitamin D deficiency in type 2 diabetic patients . J Med Sci Res. 2012, 1:32.

15. Bajaj S, Singh RP, Dwivedi NC, Singh K, Gupta A, Mathur M: Vitamin D levels and microvascular complications in type 2 diabetes. Indian J Endocrinol Metab. 2014, 18:537-41. 10.4103/2230-8210.137512

16. Mattila C, Knekt P, Männistö S, Rissanen H, Laaksonen MA, Montonen J, Reunanen A: Serum 25hydroxyvitamin D concentration and subsequent risk of type 2 diabetes. Diabetes Care. 2007, 30:2569-70. 10.2337/dc07-0292

17. Daga RA, Laway BA, Shah ZA, Mir SA, Kotwal SK, Zargar AH: High prevalence of vitamin D deficiency among newly diagnosed youth-onset diabetes mellitus in north India. Arq Bras Endocrinol Metabol. 2012, 56:423-8. 10.1590/s0004-27302012000700003

18. Bland R, Markovic D, Hills CE, Hughes SV, Chan SL, Squires PE, Hewison M: Expression of 25hydroxyvitamin D3-1alpha-hydroxylase in pancreatic islets. J Steroid Biochem Mol Biol. 2004, 89-90:121-5. 10.1016/j.jsbmb.2004.03.115 